\title{
Hierarchical Distributed Reference Counting
}

\author{
Luc Moreau \\ Department of Electronics and Computer Science \\ University of Southampton \\ Southampton SO17 1BJ, UK \\ L.Moreau@ecs.soton.ac.uk
}

\begin{abstract}
Massively distributed computing is a challenging problem for garbage collection algorithm designers as it raises the issue of scalability. The high number of hosts involved in a computation can require large tables for reference listing, whereas the lack of information sharing between hosts in a same locality can entail redundant GC traffic. In this paper, we argue that a conceptual hierarchical organisation of massive distributed computations can solve this problem. By conceptual hierarchical organisation, we mean that processors are still able to communicate in a peer to peer manner using their usual communication mechanism, but GC messages will be routed as if processors were organised in hierarchy. We present an extension of a distributed reference counting algorithm that uses such a hierarchical organisation. It allows us to bound table sizes by the number of hosts in a domain, and it allows us to share GC information between hosts in a same locality in order to reduce crossnetwork GC traffic.
\end{abstract}

\section{Introduction}

Massively distributed computing has emerged over the last few years as a potentially powerful paradigm of computation. It has taken different shapes: (i) In the I-Way experiment [9], multiple sites, communicating over wide-area networks across the American continent, are involved in a distributed computation. (ii) The World Wide Web is regarded by many as a support for planet-wide computing: amongst others, WWW programming [5], object systems on the WWW, such as Java and RMI [36], Globe [14, 18] or W3Objects [16], (iii) Software agents are autonomous programs, possibly relying on mobility $[3,22]$ to achieve a task delegated to them; they may cooperate with other agents in order to form agent societies [26]. (iv) Amorphous computing [1] involves a multitude of individuals cooperating together to provide a coherent behaviour.

For a long time, the programming language community has defended the idea that garbage collection is an automatic memory management technique that provides the programmer with a powerful abstraction of memory resources, which

Copyright 1998 by the Association for Computing Machinery, Inc. Permission to make digital or hard copies of part or all of this work for personal or classroom use is granted without fee provided that copies are not made or distributed for profit or commercial advantage and that copies bear this notice and the full citation on the first page. Copyrights for components of this work owned by others than ACM must be honored. Abstracting with credit is permitted. To copy otherwise, to republish, to post on servers, or to redistribute to lists, requires prior specific permission and/or a fee. Request permissions from Publications Dept, ACM Inc., fax +1 (212) 869-0481, or permissions@acm.org.

ISMM'98 10/98, Vancouver, Canada. eases programming. This idea has been taken on board in many languages, including the recent and widespread language Java. Distributed garbage collection, which extends the idea of automatic memory management to distributed memory, has been the focus of much attention $[17,30]$. Distributed reference counting has been a popular implementation technique of distributed garbage collection because it is simple to implement and can be nicely integrated with sequential garbage collectors [2, 24, 28, 39]. Its variant, reference listing,$[4,30,34,36]$, associates objects not only with reference counters, but also with the list of hosts that have references to them; reference listing is useful to offer some fault-tolerance.

Designing a garbage collector for massively distributed computations is, however, a challenging task. We have identified two problems that hamper the scalability of distributed garbage collection.

1. Reference listing does not scale. In the presence of massively distributed computations on the Internet, the number of hosts that have access to a given reference can become very high, which requires the garbage collector to maintain very large tables.

2. Locality is not taken into account. Distributed GC algorithms usually offer no mechanism by which sites in a same neighbourhood, e.g., two hosts in a cluster, may share information to reduce GC-related traffic with another distant host, e.g., another cluster on another continent.

The first problem is typical of any algorithm that must maintain tables of hosts; the second one is true for all reference counting algorithms that we have encountered.

Our thesis is that massively distributed computations may be conceptually organised in a hierarchy $[19,38]$ and that distributed garbage collection may rely on this organisation to overcome the two aforementioned scalability problems. An example of hierarchical organisation derived from network interconnectivity is as follows: at the lowest level, we have a COP, i.e. a cluster of PCs, connected to a laboratory network, itself in contact with a departmental backbone, which takes part in a nation-wide network, from which international connections depart. Other hierarchical organisation are conceivable, more specific to the problem being solved.

For every hierarchy, we identify a distinguished site called the gateway. The intuition of our algorithm is that the gateway acts, in the hierarchy, as the representative of the 
rest of the computation; symmetrically, it acts in the rest of the computation, as the representative of the hierarchy. For instance, if a host in a COP has access to a reference, the rest of the computation does not have to identify the precise host, but the COP gateway as holder of the reference. Similarly, a pointer being duplicated between two sites in the UK does not have to be observed by its owner in the US, as long as reference counters are correctly maintained by the UK gateway.

In this paper, we first present a flat and reference-listing variant of our algorithm for distributed reference counting and diffusion tree reorganisation (Section 2). We then convey the intuition of a hierarchical organisation (Section 3). Afterwards, we illustrate the design of hierarchical reference counting by several scenarios (Section 4). This is followed by a formal presentation of the algorithm (Section 5) and some implementation issues (Section 6). The paper ends by a discussion of related work (Section 7) and a conclusion (Section 8).

Terminology This algorithm has been designed as part of NeXeme [25], a distributed implementation of Scheme, based on the message-passing library Nexus [10]. In NeXeme, computations can proceed in different memory spaces, called sites. As in Nexus, there is a notion of global pointer $G P$ which is a first-class name for an object; a $G P$ specifies a destination to which a communication can be directed via a form of remote method invocation. As far as garbage collection is concerned, $G P$ s are references to possibly remote objects. In addition, NeXeme provides a function owner that returns the site that owns the object at which a GP is pointing.

\section{Flat Distributed Reference Counting}

We previously sketched a new algorithm for distributed reference counting [25]. We formalised this algorithm and proved its safety and liveness [24]. In this section, we briefly explain a variant of this algorithm that uses reference listing $[4,30]$; we also discuss some of its problems if used in massively distributed computations.

Each site owns two tables called Receive and Send tables, noted Rec_T and Send_T in the algorithm. A Sendtable records triples of information: the global pointers that were sent to remote sites, the sites where they were sent to, and the number of times they were sent; according to the terminology of $[4,30]$, a Send-table maintains a reference listing. Symmetrically, a Receive-table also records triples of information composed of the global pointers that were received from remote sites, the sites that emitted them, and the number of times they were received; in addition, we assume that a GP constructed (and therefore owned) by a site is entered in its Receive table.

Figure 1.1 displays the situation where a global pointer $G P$, owned by $s_{1}$, is copied from $s_{1}$ to $s_{2}$, which we model by the message $C O P Y\left(s_{1}, s_{2}, G P\right)$. If the $G P$ is copied for the first time, a new entry is created in the Send-table of $s_{1}$ for $G P$, the destination $s_{2}$, and the initial value 1 ; for every new copy towards $s_{2}$, the counter is incremented. Symmetrically, a GP received by $s_{2}$ from its owner $s_{1}$ is entered in the Receive-table; multiply receiving a $G P$ from $s_{1}$ increments the counter.

As the Send-table is implemented as a root of the local garbage collector, the presence of $G P$ in the Send-table prevents its space to be reclaimed on $s_{1}$. When $G P$ becomes garbage on $s_{2}, G P$ is removed from the Receive-table of $s_{2}$; then, a decrement message $D E C\left(s_{2}, s_{1}, G P, s_{2}\right)$ is sent from $s_{2}$ to $s_{1}$, which in turn removes the entry in the Send-table of $s_{1}$, as displayed in Figure 1.2.

The novelty of our algorithm is exhibited in Figure 1.3, when a site $s_{2}$, which does not own a $G P$, sends a copy to a third site $s_{3}$. $\quad$ (i) An entry for $G P$ and $s_{3}$ is added to the Send-table of $s_{2}$. (ii) When $G P$ is received by $s_{3}$ for the first time, an entry is added for $G P$ in its Receive-table. However, the entry records $s_{1}$, the owner of the $G P$, and not $s_{2}$, the $G P$ 's emitter. (iii) A new message $I N C_{-} D E C\left(s_{3}, s_{1}, G P, s_{2}\right)$ is sent from $s_{3}$ to the owner $s_{1}$, to inform the owner of the arrival on $s_{3}$ of a $G P$ originating from $s_{2}$ (Figure 1.3). (iv) When receiving the $I N C \_D E C$ message, the owner $s_{1}$ adds a new entry for $G P$ and $s_{3}$ in its Send-table, and then sends a $D E C\left(s_{1}, s_{2}, G P, s_{3}\right)$ message to $s_{2}$. $\quad(v)$ The decrement message sent to $s_{2}$ decrements the entry in its Send-table, and removes the entry because it becomes null.

The effect of the $I N C \_D E C$ message (followed by the $D E C$ message) is to reorganise the diffusion tree of $G P$. GP was diffused from $s_{1}$ to $s_{2}$ and then to $s_{3}$, but the tables are now recording that two copies of $G P$ owned by $s_{1}$ exist on $s_{2}$ and $s_{3}$. The benefit of this reorganisation is that if $G P$ becomes garbage on $s_{2}$, its space can be reclaimed, whereas in Piquer's indirect reference counting [29], a zombie pointer would have to be maintained on $s_{2}$ as long as $G P$ is used on $s_{3}$. Note that the algorithm correctness relies on in-order message delivery; indeed, it is essential to prevent a $D E C$ message from overtaking an $I N C \_D E C$ message, as this may result in an undesirable object reclaiming.

Further copying of $G P$ from $s_{2}$ to $s_{3}$, which do not own $G P$, increase the respective Send and Receive tables (Figure 1.5). Note that it is no longer required to involve the owner with an $I N C \_D E C$ message, because this only has to be performed the first time the $G P$ is received. At any time, a $D E C$ message may be sent to erase those entries and restore the system in the situation of Figure 1.4.

Thanks to this reorganisation mechanism, this algorithm is able to avoid zombie pointers resulting from computations jumping from node to node. This algorithm however suffers from some defects if used in the context of massively parallel computations [9, 13, 14].

1. Reference listing does not scale. The reference listing method requires Send-tables to record all the sites that have access to a given $G P$. In the presence of the Internet, this may potentially imply very large tables.

2. The algorithm does not take locality into account. In Figures 1.3 and 1.4, let us assume that sites $s_{2}$ and $s_{3}$ are connected via fast communication lines, and that communication with $s_{1}$ is slow. The technique to reorganise diffusion trees may force a communication with a site with slow communication. Similarly, in Figure 1.5 , sites $s_{2}$ and $s_{3}$ may be poorly connected and $D E C$ messages from $s_{3}$ could be grouped with other messages from sites in the neighbourhood of $s_{3}$. The lack of locality awareness also hampers the scalability of other algorithms, such as $[4,28,36]$.

In the next section, we present a hierarchical organisation of sites that allows us to avoid these two problems. 

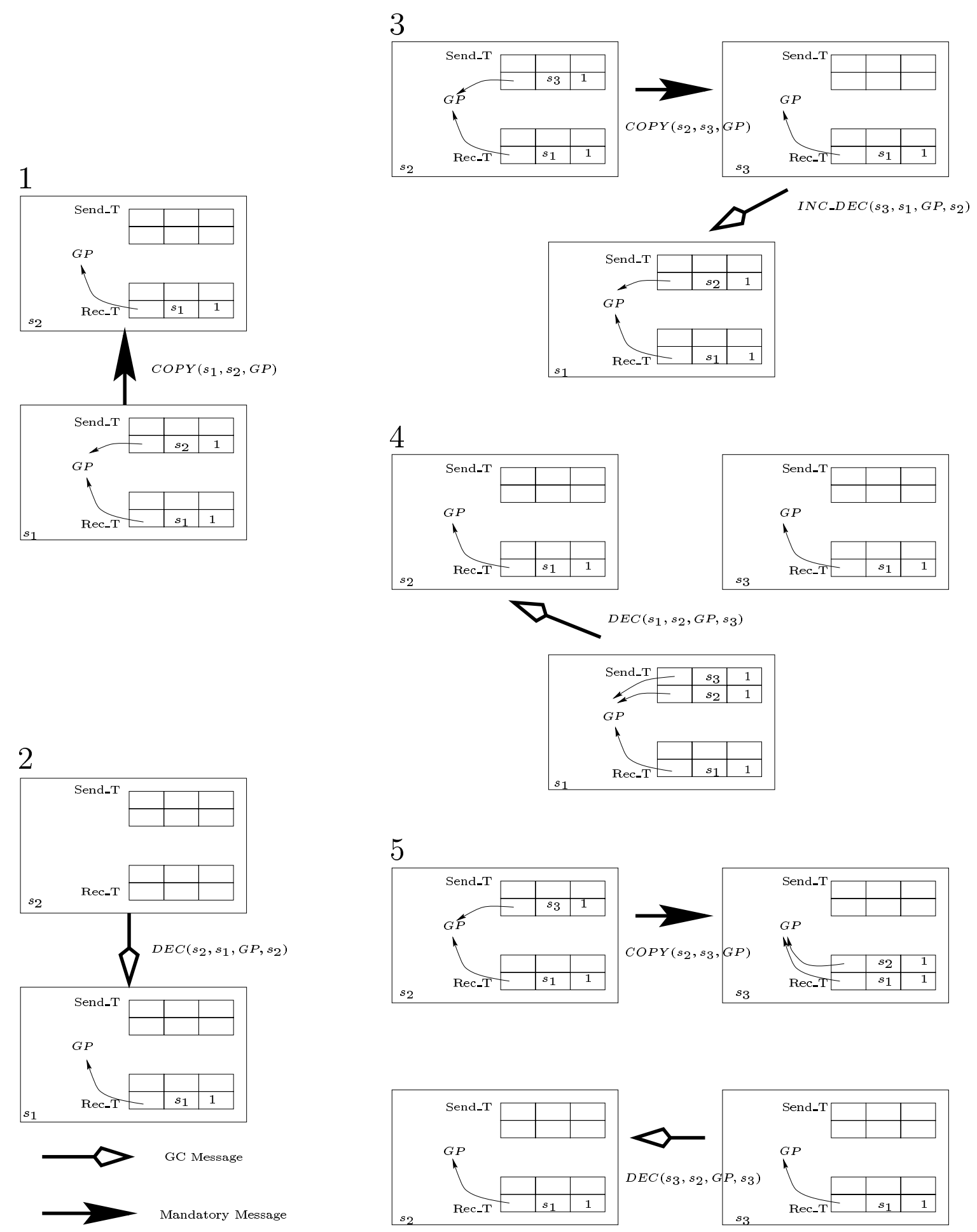

Figure 1: Flat Distributed Reference Counting 


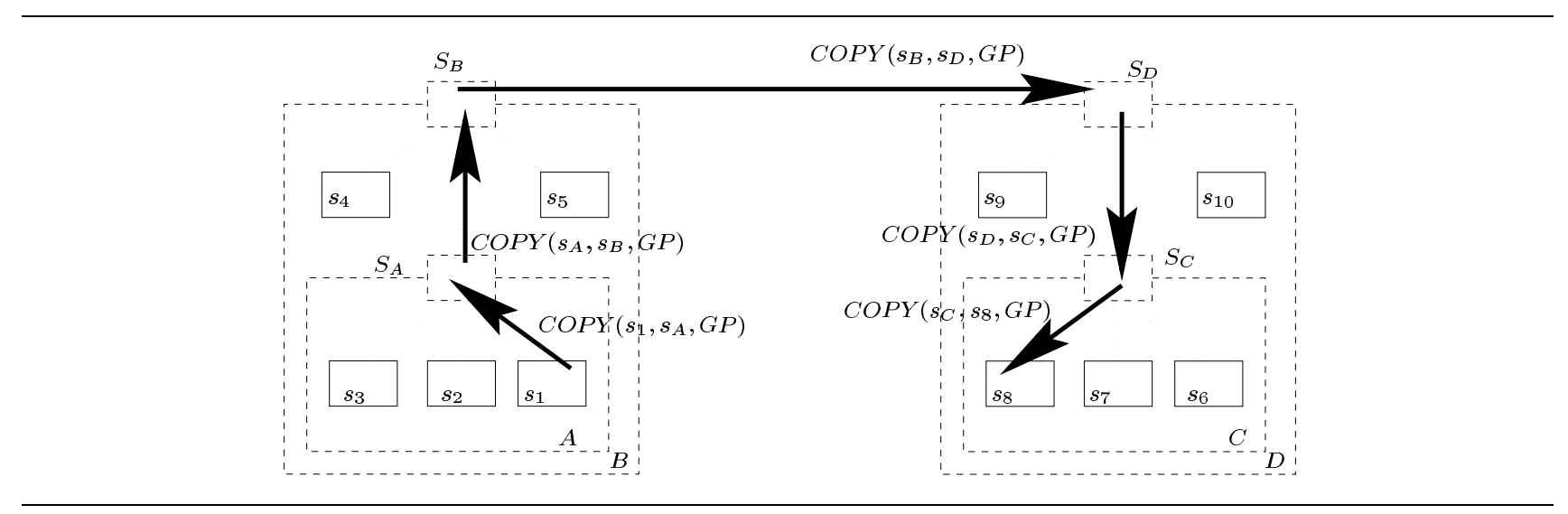

Figure 2: Hierarchical Message Forwarding

\section{Hierarchical Organisation}

Sites that are in the same proximity or in a same logical organisation are said to be grouped in domains; for instance, COPs, i.e. clusters of PCs, or NOWs, i.e. networks of workstations, may be regarded as domains. As domains are designed to follow a hierarchical organisation, domains are defined as sets of sites or subdomains. Sites (or subdomains) that belong to a domain are said to be siblings. We associate each domain with a distinguished site that acts as a gateway to the parent domain. For instance, in Figure $2, s_{1}, s_{2}, s_{3}$ are siblings and belong to a same domain $A$, with gateway $s_{A}$; sites $s_{4}, s_{5}, s_{A}$ are siblings and belong to domain $B$ whose gateway is $s_{B}$.

For garbage collection purposes, we regard the gateway as a key component of a domain: (i) The gateway $s_{A}$ of domain $A$ acts as the representative in domain $B$ of all the sites of domain $A$. In other words, elements of domain $B$ are only aware of the existence of $s_{A}$ and not of its children sites $s_{1}, s_{2}, s_{3}$. (ii) Symmetrically, the gateway $s_{A}$ of domain $A$ acts as the representative in domain $A$ of all the sites that do not belong to domain $A$. In other words, sites of $A$ are only aware of their sibling sites and of $s_{A}$ which acts as a proxy for all the other sites.

We want to use this hierarchical organisation in order to guarantee that: (i) reorganisation as in Figure 1.3 can be hidden by a domain gateway, (ii) table sizes for a given $G P$ are bounded by domain sizes. Figure 2 presents a conceptual way of implementing such a hierarchical organisation: gateways between domains could also act as message forwarders. For instance, in order to send a message from $s_{1}$ to $s_{8}, s_{1}$ sends it to the gateway $s_{A}$ because $s_{8}$ is outside domain $A$, which in turn forwards it to $s_{B}$ for the same reason, etc.

In practice such a solution is not acceptable because it potentially delays the sending of messages as it involves several domain gateways in message forwarding ${ }^{1}$. However, from a theoretical point of view, this solution is suitable because a $G P$ copied from $s_{1}$ to $s_{A}$ would create an entry for $s_{A}$ in the Send-table of $s_{1}$, and reciprocally for the Receive-table

${ }^{1}$ We assume here that we are using a network layer, such as TCP/IP, which already efficiently performs routing between networks. Every GC gateway acting as a forwarder would introduce delays. Note also that hierarchical domains may also be different from the network organisation. of $s_{A}$. Such a property would also hold for every gateway, which would guarantee that table sizes are bounded by domain sizes.

In the next Section, we present an extension of the flat GC that does not increase the cost of sending mandatory messages, but maintains a hierarchical organisation as we just described.

\section{Hierarchical Distributed Reference Counting}

As in every garbage collector design, it is essential to minimize the impact of garbage collection activity on mandatory computation. In particular, remote method invocation, which copies global pointers, should be executed as efficiently as possible. Therefore, instead of hierarchical message forwarding of Figure 2, we prefer the organisation of Figure 3 , where we see that the mandatory message is directly sent from $s_{1}$ to $s_{8}$, but a conceptual hierarchy is kept for GC purposes. Two new messages, introduced to inform gateways of cross-domain messages, are asynchronously sent to gateways of the hierarchy; they are defined in terms of three sites $s_{1}, s_{2}, s_{3}$ :

- With DOM_SEND $\left(s_{1}, s_{2}, G P, s_{3}\right.$ ), site (or subdomain) $s_{1}$ informs its gateway $s_{2}$ that $G P$ was sent to $s_{3}$ belonging to another domain.

- With DOM_RECV $\left(s_{1}, s_{2}, G P, s_{3}\right.$ ), site (or subdomain) $s_{1}$ informs its gateway $s_{2}$ that $G P$ was received from $s_{3}$ belonging to another domain.

In Figure 3, DOM_SEND and DOM_RECV messages are repeatedly sent till they respectively reach $s_{B}$ and $s_{D}$, such that $s_{B}$ is an ancestor of $s_{1}, s_{D}$ is an ancestor of $s_{8}$, and $s_{B}$ is sibling of $s_{D}$.

When messages are exchanged within a domain, the flat garbage collection algorithm of Figure 1 is used. If global pointers are sent to or received from sites belonging to other domains, then Figure 4 summarises the hierarchical protocol.

In Figure 4.1, site $s_{2}$ sends a copy of $G P$ to $s_{3}$, a site outside the domain $A$. A new entry is added to the Sendtable of $s_{2}$, with $G P$ and the gateway $s_{A}$, as if the message had been routed via the gateway $s_{A}$. In addition, a message $D O M_{-} S E N D$ is sent to the gateway, which acts as if it was forwarding the message. Gateways also maintain Receive 


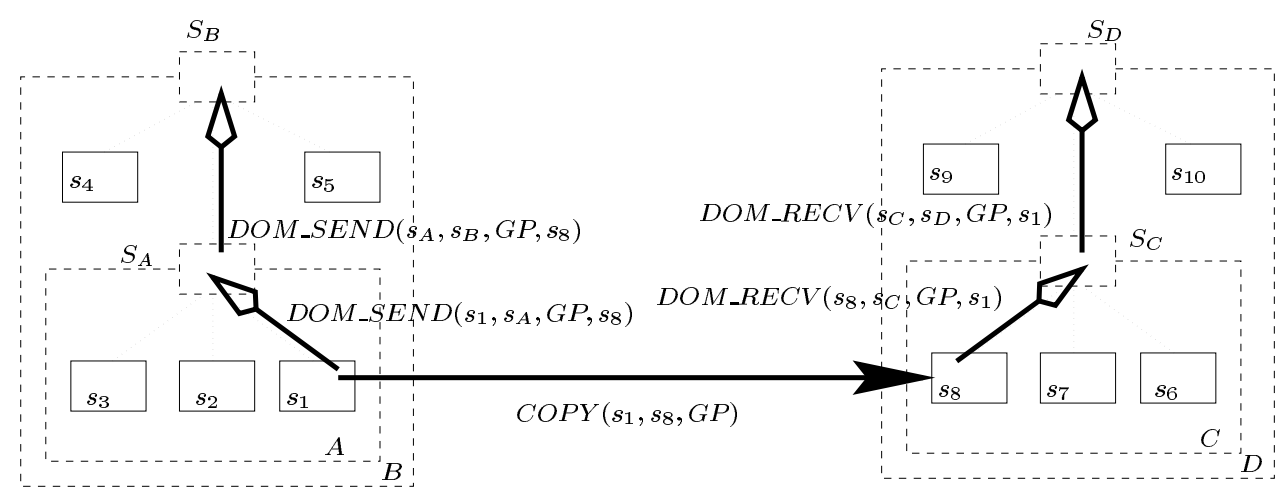

Figure 3: Hierarchical Update of Tables

and Send tables: (i) Within domain $A$, the flat GC algorithm applies. If this is the first time that $s_{A}$ receives a copy of $G P$, and if $s_{1}$ owns $G P$, then $I N C \_D E C$ and $D E C$ messages are sent in the usual triangular mode, and the Receivetable of $s_{A}$ records that $G P$ comes from $s_{1}$. (ii) If $s_{3}$ (or one of its gateways) is a sibling of $s_{A}$, then the Send-table entry of $s_{A}$ records this gateway. Otherwise, if $s_{3}$ (or one of its gateways) is not a sibling of $s_{A}$, then the same mechanism applies recursively, and $s_{A}$ sends a DOM_SEND message to its gateway, etc. Figure 3 illustrates a succession of such $D O M \_S E N D$ messages. A DOM_SEND message is successively sent to gateways of the hierarchy till it reaches a gateway $s_{B}$ (in Figure 3), which is a sibling of a gateway $s_{D}$, itself an ancestor of the GP receiver $s_{8}$.

Figure 4.2 describes the symmetric situation where a site $s_{1}$ receives a copy of a $G P$ from $s_{3}$ outside the domain $A$. The system should behave as if $G P$ had been received from the gateway $s_{A}$. Hence, a DOM $R E C V$ is sent to the gateway $s_{A}$, which implies that the Send-table of $s_{A}$ records that $G P$ is sent to $s_{1}$, and symmetrically for the Receive-table of $s_{1}$. If it is the first time that $G P$ is received by $s_{1}$ and if $G P$ is owned by $s_{2}$, a sequence of $I N C \_D E C$ and $D E C$ messages is required as in the flat GC algorithm ${ }^{2}$. If $s_{3}$ (or one of its gateways) is not a sibling of $s_{A}$, then we proceed recursively as illustrated in Figure 3. A DOM_RECV message is successively sent to gateways of the hierarchy till it reaches a gateway $s_{D}$ (in Figure 3 ), which is a sibling of a gateway $s_{B}$, itself an ancestor of the GP emitter $s_{1}$.

In summary, gateways act as the Receive- and Send- tables of domains. The DOM_SEND or DOM_RECV messages consist of incrementing the Send or Receive tables in the gateway, respectively, possibly entailing a triangular reorganisation as in the flat version of the algorithm.

\section{The Algorithm}

We formalise the algorithm using an abstract machine, called the HGC machine, whose configuration is defined at

\footnotetext{
${ }^{2}$ The $D O M_{-} R E C V$ message to $s_{A}$ is in competition with the $D E C$ message which respectively increments and decrements the counter for $G P$ in the Send-table of $s_{A}$. Even though this may decrement the counter before it is increased, and hence result in a temporary negative value, this has no consequence on the correctness because the $D E C$ message sent from the owner has previously increased a counter on the owner, hereby preventing undesirable reclaiming.
}

the top of Figure 5. A configuration $\mathcal{H}$ is a tuple composed of a set of sites, a set of global pointers, Receive and Send tables for every site, and a bag of messages. Receive and Send tables are functions which for a site $s_{1}$, a $G P$, a site $s_{2}$ respectively return the number of times $G P$ was received by $s_{1}$ from $s_{2}$, or sent from $s_{1}$ to $s_{2}$. The bag of messages denotes messages that are in transit in the system, i.e. already posted but not yet handled.

We write owner $(G P)$ to denote the site that owns $G P$. Initially, all table entries are zero, except for hosts that own GPs:

$$
\begin{aligned}
\text { send_T }\left(s_{1}, G P, s_{2}\right) & =0, \forall s_{1}, s_{2}, G P, \\
r e c \_T(s, G P, s) & =1 \text { if owner }(G P)=s \\
r e c \_T\left(s_{1}, G P, s_{2}\right) & =0, \forall s_{1}, s_{2}, G P, \text { otherwise. }
\end{aligned}
$$

We allow HGC-configurations to perform four transitions. Let $\mathcal{H}_{1}$ be $\langle\mathcal{S}, \mathcal{G}$, send_T, rec_T, $\mathcal{M}\rangle$, and let us assume that $s_{1}, s_{2}, s_{3} \in \mathcal{S}, G P \in \mathcal{G}, m \in \mathcal{M}$, then:

$$
\begin{array}{rrrr}
\mathcal{H}_{1} \Rightarrow & \text { MAKE_COPY }\left(s_{1}, s_{2}, G P\right) \Rightarrow \mathcal{H}_{2} & \text { (make-copy) } \\
\mathcal{H}_{1} \Rightarrow \operatorname{RECEIVE}(m) \Rightarrow \mathcal{H}_{2} & \text { (receive) } \\
& \text { if } m=\operatorname{DEC}\left(s_{1}, s_{2}, G P\right), \text { then } & \\
& \operatorname{INC} \operatorname{DEC}\left(s_{1}, s_{2}, G P, s_{3}\right) \notin \mathcal{M}, \forall s_{3} & \\
\mathcal{H}_{1} \Rightarrow \operatorname{RELEASE}\left(s_{1}, G P\right) \Rightarrow \mathcal{H}_{2} & \text { (release) } \\
\mathcal{H}_{1} \Rightarrow \operatorname{DELETE}\left(s_{1}, s_{2}, G P\right) \Rightarrow \mathcal{H}_{2} & \text { (delete) }
\end{array}
$$

The configuration transformers MAKE_COPY, RECEI$V E, R E L E A S E$ and DELETE are defined in Figures 5 and 6. In Figure 5 , if $\mathcal{H}_{1}=\langle\mathcal{S}, \mathcal{G}$, send_T, rec_T, $\mathcal{M}\rangle$, post $(m)$ denotes the configuration $\mathcal{H}_{2}$ such that $\mathcal{H}_{2}=\langle\mathcal{S}, \mathcal{G}$, send_T, rec $T, \mathcal{M} \cup\{m\}\rangle$. Similar notational conventions are used for assignments to Send and Receive tables. In the rules above, let us note the side-condition of (receive), which may be implemented by in-order message delivery. We also assume that transitions are executed atomically.

In addition, in order to model the hierarchical domain organisation, we use the following relations:

- parent $(x, y)$ : node $y$ is parent of node $x$.

- descendant $(x, y)$ : node $y$ is a descendant of node $x$, if $x=y$ or $\exists z$,parent $(z, x) \wedge$ descendant $(z, y)$. 


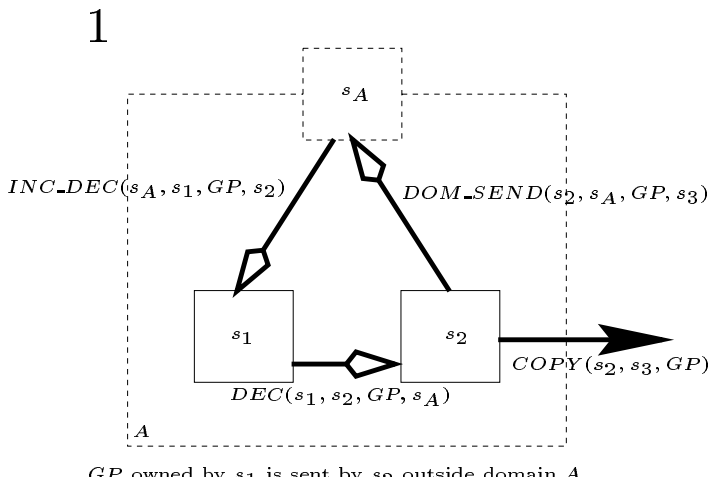

$G P$ owned by $s_{1}$ is sent by $s_{2}$ outside domain $A$

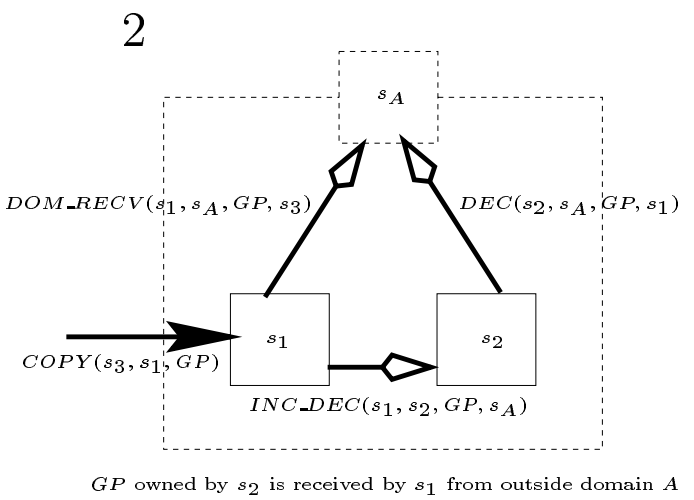

Figure 4: Cross-Domain Pointer Copying

- $\operatorname{sibling}(x, y)$ : node $y$ is a sibling of node $x$, if $\exists z$, $\operatorname{parent}(x, z)=\operatorname{parent}(y, z)$.

- $\operatorname{proxy}(x, y)$ : the representative of $y$ in the domain of $x$. For the time being, we consider a static hierarchical organisation; the proxy relation is defined as follows.

$$
\begin{gathered}
\operatorname{proxy}(x, y) \\
=\left\{\begin{array}{cc}
y & \text { if } \operatorname{sibling}(x, y) \\
z & \text { if } \neg \operatorname{sibling}(x, y) \wedge \neg \operatorname{descendant}(x, y) \\
z & \text { if } \wedge \operatorname{parent}(x, z) \\
& \wedge \operatorname{paring}(x, y) \wedge \operatorname{descendant}(z, y)
\end{array}\right.
\end{gathered}
$$

Let us first consider the particular case where all sites belong to the same domain, which means that $\operatorname{sibling}(x, y)$ holds and that $\operatorname{prox} y(x, y)=y$ for any $x, y$. In such a situation, Figure 5 describes the algorithm for "flat" garbage collection.

Rule (make-copy) associated with configuration transformer $M A K E \_C O P Y$ models the actions that site $s_{1}$ has to take before sending a copy of GP to $s_{2}$. The send_T is updated and a $C O P Y$ message is posted.

Rule (receive) and its associated configuration transformer RECEIVE describe how messages are received. When $s_{2}$ receives a $C O P Y$ message from $s_{1}$, a triangular reorganisation is initiated if it is the first time $G P$ is received, i.e. the entry in the receive table for the owner $s_{g}$ is empty. The triangular reorganisation involves sending an $I N C \_D E C$ message to the owner, which is followed by a $D E C$ message.

An entry in the receive table pointing at a site that is not the owner of a GP may always be cleared by rule (release), which sends a decrement message to the site. Finally, a local garbage collector that proves that a $G P$ has become garbage on site $s$ initiates the transition (delete); such a transition can only be fired if Receive and Send tables (except for the owner $s_{g}$ ) are empty, and it results in a $D E C$ message sent to the owner.

If the sites involved in the transitions do not belong to the same domain, rules of Figure 5 remain still applicable, but are now involving proxies of the sites. For instance, receiving a $C O P Y$ message from $s_{1}$, which does not belong to the same domain as $s_{2}$, potentially results in a triangular reorganisation with $s_{p}$ and $s_{g}$, respectively proxies of $s_{1}$ and the owner (cf. Figure 4.2). In addition, a DOM_RECV message is sent, when $s_{2}$ is not sibling with (the proxy of) $s_{1}$.

Figure 6 displays how the two messages for hierarchical GC are handled. Receiving a message $D O M_{-} R E C V\left(s_{1}, s_{2}, G P, s_{3}\right)$ is similar to receiving a message $\operatorname{COPY}\left(s_{3}, s_{2}, G P\right)$. The only difference is that the gateway $s_{2}$ has to act $a s$ if it was forwarding the message originating from $s_{3}$ to $s_{1}$ (as in Figure 2). As a result, the entry for $s_{1}$ in the send_T of $s_{2}$ has to be incremented. 


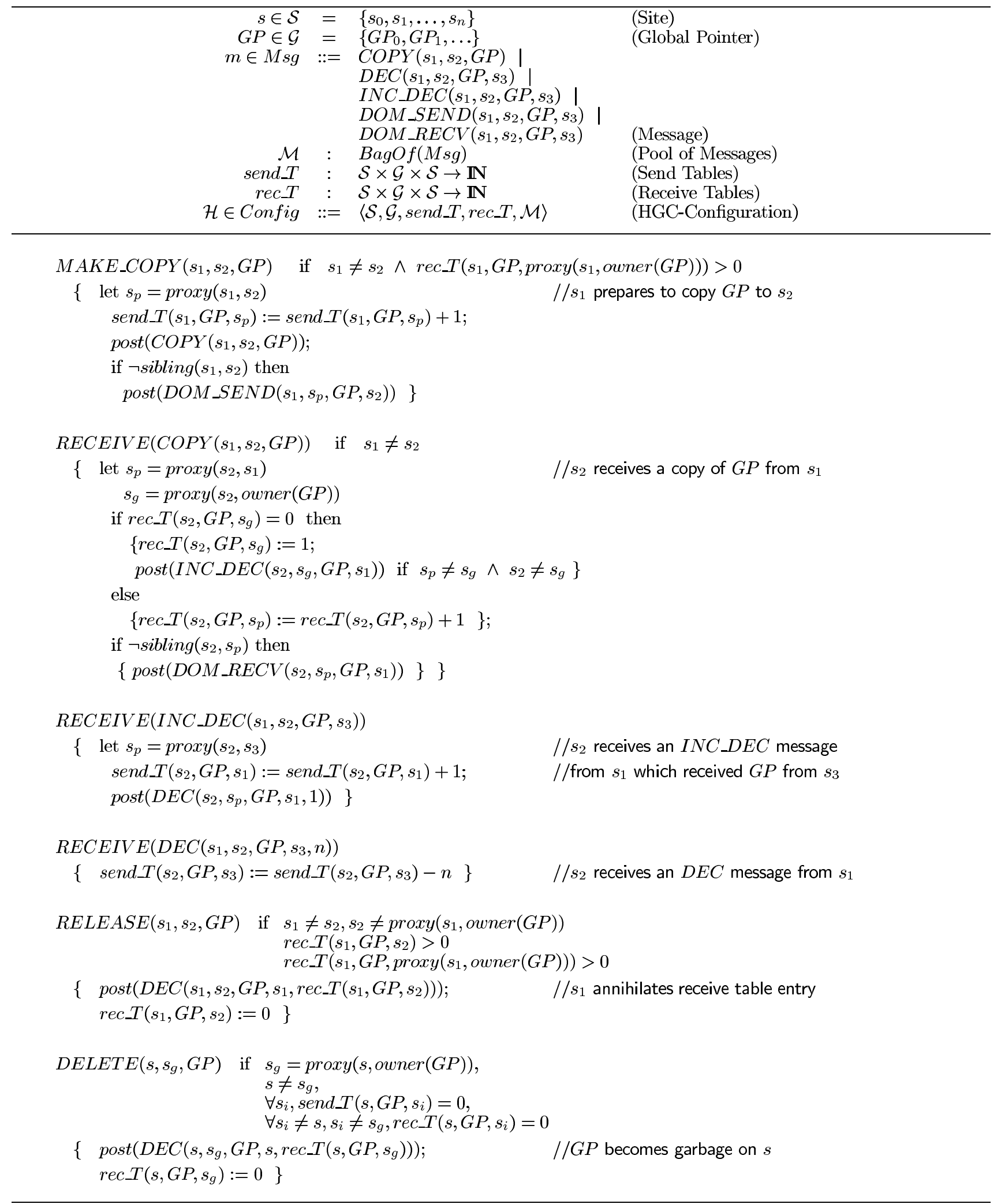

Figure 5: Flat Garbage Collection 


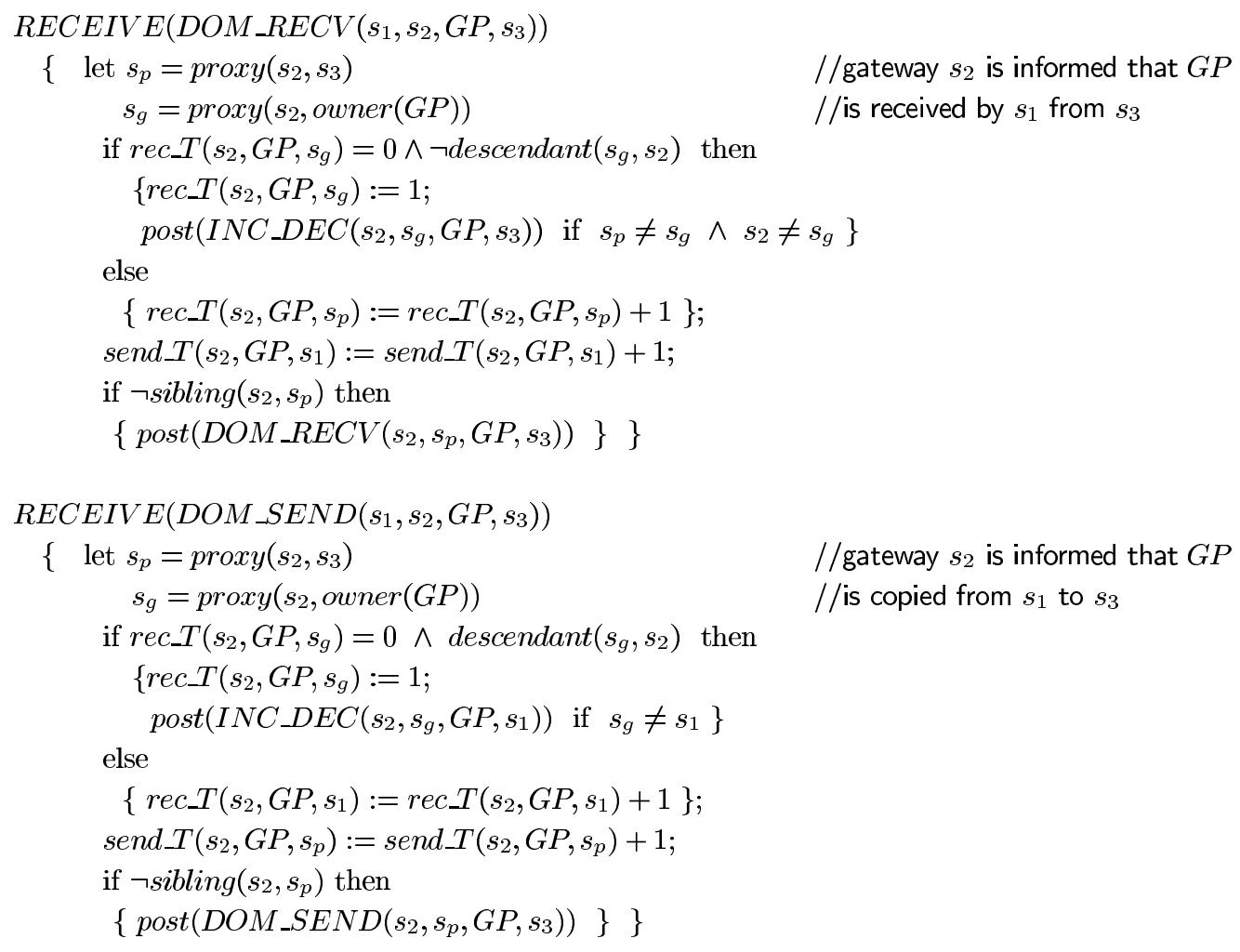

//gateway $s_{2}$ is informed that $G P$

$/ /$ is received by $s_{1}$ from $s_{3}$

//gateway $s_{2}$ is informed that $G P$

$/ /$ is copied from $s_{1}$ to $s_{3}$

Figure 6: Hierarchical Garbage Collection

Conceptually, when a gateway $s_{2}$ receives a message $D O M_{-} S E N D\left(s_{1}, s_{2}, G P, s_{3}\right)$ from $s_{1}$, it must act as if it was forwarding the message to its destination $s_{3}$. Therefore, the receive table records the arrival of $G P$ from $s_{1}$ (or $s_{g}$ via the usual triangular reorganisation). In addition, the entry for the proxy of $s_{3}$ is incremented in the Send-table of $s_{2}$.

Our hierarchical organisation solves the problem of the flat GC algorithm:

- Locality: Gateways are hiding reorganisations within their domains. For instance, the copy of $G P$ owned by a site outside the domain results in a triangular exchange as described by Figure 7, where the gateway acts as a representative for the $G P$ 's owner.

In addition, as the gateway $s_{A}$ of Figure 7 "centralises" GC information about $G P$ for domain $A$, we are able to send a decrement message to its owner only when $G P$ has become garbage on all sites of domain $A$. Such sharing of information in a domain allows us to reduce GC traffic across domains.

- Table Size: per global pointer, a table now has a maximum number of entries given by the size of the domain. Indeed, every access to a Send-Table (and similarly for a Receive-Table) in the algorithm of Figures 5 and 6 is of the form send_T $\left(s_{1}, G P, s_{x}\right)$, where $s_{x}$ was defined as $\operatorname{proxy}\left(s_{1}, s_{3}\right)$, for any site $s_{3}$. So, entries in SendTables always refer to sites in the same domain.

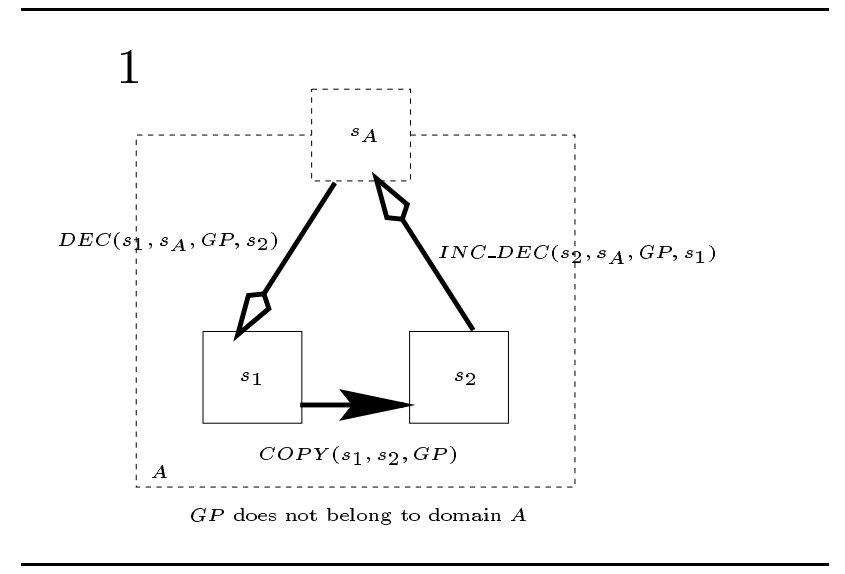

Figure 7: Inside Domain Triangular Reorganisation

\section{Implementation}

This distributed GC algorithm has been designed as part of NeXeme [25] a distributed extension of Scheme based on the message-passing library Nexus [10]. The flat algorithm has been fully implemented and tested, but (at the time of writing), the hierarchical implementation is still in progress.

An important aspect of the implementation is the design of an efficient function sibling. Currently, we define a hier- 
archical pointer as a structure composed of a global pointer and an access path, which is a datastructure representing the path of its owner in the hierarchy. By default, the access path is set to nil, which means that the hierarchical pointer has not exited its domain. Every time a hierarchical pointer is serialised, we have to compare its access path with the access path of the message destination. If the access paths are equal, then the hierarchical pointer is being sent to its domain. Otherwise, from the common path prefix, one can derive the gateways to which the DOM_SEND and DOM_RECV messages must be sent.

For the time being, gateways are built as distinguished processes. We are investigating how to implement them as regular nodes, which could also take part in the computation. So far, we have considered a static hierarchy. We are also studying ways of specifying the hierarchy dynamically and of changing gateways at runtime according to the load of the system.

We have presented here an abstract algorithm, which can be optimised in several ways. (i) Several DOM_SEND (or DOM_RECV) could be merged together, in the same spirit as $D E C$ messages that contain a counter value. (ii) One message could be avoided in Figure 4.2 by recognising this particular situation, to the detriment of algorithm readability; for instance, the $D O M_{-} R E C V$ and $D E C$ messages could be combined in a new message that would follow the INC_DEC message. (iii) We can design different strategies for sending GC messages. For the time being, a regular sites sends GC messages as soon as it becomes idle. On the other hand, gateways infrequently send messages to their siblings in order to avoid cross-domain traffic.

\section{Related Work}

Reference-counting garbage collection was initially developed for uniprocessor systems [6]. Its principle is as follows: every time a pointer is copied or deleted, a reference counter is respectively incremented or decremented. It might seem that this algorithm can be extended straightforwardly to distribution by using two control messages INC and $D E C$ that act on the reference counter residing on the owner of the pointer. Unfortunately, non-causal message delivery may reset the counter even though remote references may still be active. Correct solutions to this problem have been proposed, including weighted reference counting $[2,8,39]$ and its optimised version [7], generational reference counting [12], indirect reference counting [28, 29].

Other tripartite exchange of messages including the site that emitted a $G P$, the receiver of the $G P$ and its owner can be found in the literature, in particular by Lermen and Maurer [21, 37], and by Birrel et al. [4]. Our algorithm differs from theirs by the direction in which messages are exchanged and because our solution only requires a tripartite exchange the first time a $G P$ is received. Intuitively, our solution preserves causality because the owner sends a $D E C$ message only after having processed an $I N C \_D E C$ message which has increased a reference counter.

However, our algorithm has another major benefit as it is able to reorganise diffusion trees: when GC messages are all processed, the diffusion tree is completely flattened, and every site owning a GP directly "depends" from its owner. In the presence of mobile computations jumping from site to site, this allows sites to reclaim the space that was occupied by a mobile program, hereby avoiding zombie refer- ences as in indirect reference counting [28]. To the best of our knowledge, Shapiro, Dickman, and Plainfossé [34, 35] were the first to address the issue of short-cutting chains of pointers. They regard migration as a primitive notion to be supported by the GC; in this paper, we do not deal with migration, however, we have showed that support for mobility could be added as an extra layer, like a library, on top of the current garbage collection algorithm [24].

The distributed collector of Java with Remote Method Invocation [36] is derived from Birrel's network objects [4]. In addition, Java uses a mechanism of lease, by which sites having pointer copies are forced to regularly renew their lease. Such a mechanism supports fault-tolerance and could also be implemented with our algorithm.

Let us note that none of the previously mentioned algorithms is based on a hierarchical organisation as presented in this paper. Therefore, those using reference listing $[4,35,36]$ potentially have to manage very large tables in Internetwide computation. Furthermore, they are not able to share garbage collection information within a neighbourhood, which prevents them from optimising GC information transfer on a local basis, such as per cluster or per network.

We are accustomed to hierarchical memories in uniprocessor systems and memory management has been studied in this particular case [40]. We can regard our schema as a hierarchical organisation of a distributed memory. Other hierarchical organisations have been brought forward. Lang, Queinnec, and Piquer "Garbage collecting the World" uses a hierarchy of nodes that are willing to cooperate for garbage collection; such a similar approach is also adopted in [38] in order to provide scalability. Queinnec [31] also suggests to cluster sites so that they can present a single clock to the rest of the world; clocks are used to provide a causally-coherent distributed memory.

Rodrigues and Jones [32] dynamically identify groups of processes that will collaborate to reclaim distributed cyclic garbage. Their groups provide locality as communication related to the garbage collection activity is only necessary between members of the group. The same authors [33] also explain how groups that have independently initiated a collection on the same cyle may merge together. Maheshwari and Liskov [23] use back tracing [11] to determine if an object is garbage. Back tracing as opposed to forward tracing preserve locality of the tracing process.

Reference counting garbage collection is only able to reclaim acyclic data structures. Other algorithms may be combined with ours in order to collect cycles, such as Le Fessant, Piumarta, and Shapiro's [20], Rodrigues and Jones' [32], or Lang, Queinnec and Piquer's [19]. The latter seems to be particularly appropriate because it also relies upon a hierarchical organisation of sites that cooperate to eliminate cycles between themselves. Gateways in our algorithm contain Send and Receive tables for a domain and can be used to perform a collaborative garbage collection of the domain. The distributed variant of the Train GC [15] is also able to collect cycles; it combines a reference-counting style pointer-tracking mechanism with a substitution protocol.

\section{Conclusion}

We have presented a hierarchical organisation for distributed reference counting. Such an approach is particularly suitable for Internet-wide programming because it is able to abstract a whole domain of sites by a single host, which acts as its 
representative for garbage collection purposes. Such a hierarchical schema allows us to give bounds on the size of tables involved in reference listing and to reduce cross domain GC traffic.

This algorithm is being implemented as part of NeXeme, a distributed implementation of Scheme [25], offering some support for mobile computations [24] and distributed resource control [27]. We foresee two other applications for this algorithm. First, in [26], we associate reference counters with WWW documents, in order to build an agent architecture that offers link integrity in a publishing environment; the new reference counting algorithm may be used to improve scalability of the system. Second, in [27], we present a distributed model of resource control, suitable for agentstyle applications. We believe that the present algorithm can be applied to resource control in order to facilitate resource management among sites in a same locality.

\section{Acknowledgement}

This research was supported in part by the Engineering and Physical Sciences Research Council, grant GR/K30773. We are grateful to Stuart Maclean, Danius Michaelides, Christian Queinnec, and the anonymous referees for their comments.

\section{References}

[1] Harold Abelson, Thomas F. Knight, Gerald Jay Sussman, and friends. Amorphous Computing Manifesto. Technical report, MIT, 1996. available from http://www-swiss.ai.mit.edu/ switz/amorphous.

[2] David I. Bevan. Distributed Garbage Collection using Reference Counting. In PARLE Parallel Architectures and Languages Europe, volume 259 of Lecture Notes in Computer Science, pages 176-187. Springer-Verlag, June 1987.

[3] Krishna Bharat and Luca Cardelli. Migratory Applications. In Mobile Object Systems: Towards the Programmable Internet, pages 131-149. Springer-Verlag, April 1997. Lecture Notes in Computer Science No. 1222.

[4] Andrew Birrell, David Evers, Greg Nelson, Susan Owicki, and Edward Wobber. Distributed Garbage Collection for Network Objects. Technical Report 116, Digital Systems Research Center, 130 Lytton Avenue, Palo Alto, CA 94301, December 1993.

[5] Luca Cardelli and R. Davies. Service Combinators for Web Computing. In Usenix Conference on Domain Specific Languages DSL97, Santa-Barbara, California, October 1997.

[6] George E. Collins. A Method for Overlapping and Erasure of Lists. Communications of the ACM, 3(12):655657, December 1960.

[7] Peter Dickman. Optimising Weighted Reference Counts for Scalable Fault-Tolerant Distributed ObjectSupport fsystems, 1992.

[8] Ian Foster. A Multicomputer Garbage Collector for a Single-Assignment Language. Intl J. of Parallel Programming, 18(3):181-203, 1989.
[9] Ian Foster. High-Performance Distributed Computing: the I-WAY Experiment and Beyond. In Euro-Par'96 Parallel Processing, volume 1123 of Lecture Notes in Computer Science, pages 3-10, Lyon, France, August 1996.

[10] Ian Foster, Carl Kesselman, and Steven Tuecke. The Nexus Approach to Integrating Multithreading and Communication. Journal of Parallel and Distributed Computing, 37:70-82, 1996.

[11] Matthew Fuchs. Garbage Collection on an Open Network. In Proceedings of International Workshop on Memory Management, volume 986 of Lecture Notes in Computer Science, Kinross, Scotland, September 1995.

[12] Benjamin Goldberg. Generational Reference Counting: A Reduced-Communication Distributed Storage Reclamation Scheme. In SIGPLAN Programming Language Design and Implemantation PLDI'89, pages 313-320, 1989.

[13] Andrew S. Grimshaw, William A. Wulf, James C. French, Alfred C. Weaver, and Paul F. Reynolds, Jr. A Synopsis of the Legion Project. Technical Report CS-94-20, Deparment of Computer Science, University of Virginia, June 1994.

[14] P. Homburg, M. van Steen, and A. S. Tanenbaum. Communicating in GLOBE: an Object-Based Worldwide Operating System. In Proc. Fifth International Workshop on Object Orientation in Operating Systems, pages 43-47, Seattle, Washington, October 1996.

[15] R.L. Hudson, R. Morrison, J.E.B. Moss, and D.S. Munro. Garbage Collecting the World: One Car at a Time. In Proceedings of OOPSLA'9'7, Atlanta, USA, 1997.

[16] D. B. Ingham, M. C. Little, S. J. Caughey, and S. K. Shrivastava. W3objects: Bringing Object-Oriented Technology to the Web. In Proc. Fourth International World-Wide Web Conference, pages 89-105, Boston, Mass., USA, December 1995.

[17] Richard Jones and Rafael Lins. Garbage Collection. Algorithms for Automatic Dynamic Memory Management. Wiley, 1996.

[18] I. Kuz, A.M. Kermarrec, M. van Steen, and H.J. Sips. Replicated Web Objects: Design and Implementation. In Proc. Fourth Annual ASCI Conference, Lommel, Belgium, June 1998.

[19] Bernard Lang, Christian Queinnec, and José Piquer. Garbage Collecting the World. In Proceedings of the Nineteenth Annual ACM SIGACT-SIGPLAN Symposium on Principles of Programming Languages, pages 39-50, Albuquerque, New Mexico, January 1992.

[20] Fabrice Le Fessant, Ian Piumarta, and Marc Shapiro. A Detection Algorithm for Distributed Cycles of Garbage. In OOPSLA'9'7 Garbage Collection and Memory Management Workshop. http://www.dcs.gla.ac.uk/ huw/oopsla97/ gc/papers.html, 1997.

[21] C.-W. Lermen and D. Maurer. A Protocol for Distributed Reference Counting. In Lisp and Functional Programming, pages 343-354, 1986. 
[22] General Magic. Telescript Technology: Mobile Agents. http://www.genmagic.com/ Telescript/Whitepapers/ wp4/whitepaper-4.html, 1996.

[23] Umesh Maheshwari and Barbara Liskov. Collecting Cyclic Distributed Garbage by Back Tracing. In Proceedings of PODC'977 Principles of Distributed Computing, 1997.

[24] Luc Moreau. A Distributed Garbage Collector with Diffusion Tree Reorganisation and Object Mobility. In Proceedings of the Third International Conference of Functional Programming (ICFP'98), Septembre 1998.

[25] Luc Moreau, David DeRoure, and Ian Foster. NeXeme: a Distributed Scheme Based on Nexus. In Third International Europar Conference (EURO-PAR'97), volume 1300 of Lecture Notes in Computer Science, pages 581590, Passau, Germany, August 1997. Springer-Verlag.

[26] Luc Moreau and Nicholas Gray. A Community of Agents Maintaining Links in the World Wide Web (Preliminary Report). In The Third International Conference and Exhibition on The Practical Application of Intelligent Agents and Multi-Agents, pages 221-235, London, UK, March 1998.

[27] Luc Moreau and Christian Queinnec. Distributed Computations Driven by Resource Consumption. In IEEE International Conference on Computer Languages (ICCL'98), pages 68-77, Chicago, Illinois, May 1998.

[28] José M. Piquer. Indirect Reference Counting: A Distributed Garbage Collection Algorithm. In Parallel Architectures and Languages Europe (PARLE'91), pages 150-165, 1991.

[29] José M. Piquer. Indirect Distributed Garbage Collection: Handling Object Migration. ACM Transactions on Programming Languages and Systems, 18(5):615647, September 1996.

[30] David Plainfossé and Marc Shapiro. A Survey of Distributed Garbage Collection Techniques. In Henry G. Baker, editor, International Workshop on Memory Management (IWMM95), number 986 in Lecture Notes in Computer Science, pages 211-249, Kinross, Scotland, 1995.

[31] Christian Queinnec. Sharing Mutable Objects and Controlling Groups of Tasks in a Concurrent and Distributed Language. In Takayasu Ito and Akinori Yonezawa, editors, Proceedings of the Workshop on Theory and Practice of Parallel Programming (TPPP'94), number 700 in Lecture Notes in Computer Science, pages 70-93, Sendai (Japan), November 1994. Springer-Verlag.

[32] Helena C. C. D. Rodrigues and Richard E. Jones. A Cyclic Distributed Garbage Collector for Network Objects. In Tenth International Workshop on Distributed Algorithms WDAG'96, number 1151 in Lecture Notes in Computer Science, Bologna, October 1996.

[33] Helena C. C. D. Rodrigues and Richard E. Jones. Cyclic Distributed Garbage Collection with Group Merger. In
Proceedings of 12th European Conference on ObjectOriented Programming, ECOOP98, Lecture Notes in Computer Science, pages 249-273, Brussels, 1998.

[34] Marc Shapiro, Peter Dickman, and David Plainfossé. Robust, Distributed References and Acyclic Garbage Collection. In Symposium on Principles of Distributed Computing, Vancouver, Canada, August 1992.

[35] Marc Shapiro, Peter Dickman, and David Plainfossé. SSP Chains: Robust, Distributed References Supporting Acyclic Garbage Collection. Rapport de Recherche 1799, INRIA-Rocquencourt, November 1992.

[36] Sun MicroSystems. Java Remote Method Invocation Specification, November 1996.

[37] Gerard Tel and Friedemann Mattern. The Derivation of Distributed Termination Detection Algorithms from Garbage Collection Schemes. ACM Transactions on Programming Languages and Systems, 15(1):1-35, January 1993.

[38] Nalini Venkatasubramanian, Gul Agha, and Carolyn Talcott. Scalable Distributed Garbage Collection for Systems of Active Objects. In Proc. 1992 International Workshop on Memory Management, pages 134147, Saint-Malo (France), September 1992. SpringerVerlag.

[39] Paul Watson and Ian Watson. An Efficient Garbage Collection Scheme for Parallel Computer Architectures. In PARLE Parallel Architectures and Languages Europe, volume 259 of Lecture Notes in Computer Science, pages 432-443. Springer-Verlag, June 1987.

[40] Paul R. Wilson. Uniprocessor Gargage Collection Techniques. In International Workshop on Memory Management, Lecture Notes in Computer Science, SaintMalo, France, September 1992. 